\title{
Electronic pre-transfusion check at the bedside: experience in a university hospital
}

\begin{abstract}
Although the current risks of viral transmission through blood components are very small, mistransfusion in which the wrong blood is transfused to the wrong patient remains the most common type of error in transfusion practice. Many studies have revealed that transfusion errors occur frequently in clinical areas, with the most common error being failure to perform the final patient identification check at the bedside. Thus, pre-transfusion check at the bedside is the most critical step for the prevention of mistransfusion. Machine-readable identification technology, especially an electronic identification system (EIS), is ideally suited to pre-transfusion check requirements. An experience at the Juntendo University Hospital (JUH) showed that the bar code-based EIS works well on a hospital-wide basis in the setting of regular allogeneic blood transfusion, preoperative autologous blood donation (PAD) and transfusion, pediatric transfusion and hematopoietic progenitor cell (HPC) infusion at the bedside. Approximately 110,000 blood components have been transfused over a 10.5-year period without a single mistransfusion. The overall compliance rate with electronic pre-transfusion check at the bedside was $98.2 \%$. Human error was the most frequent cause of errors leading to failure of the 'second' electronic pre-transfusion check. If we want to reduce the risk of mistransfusion to improve transfusion safety, we have to address the issue at the hospital level, with a system-based approach.
\end{abstract}

Keywords: transfusion safety, mistransfusion, electronic pre-transfusion check, ABO-incompatible, hemovigilance, oximetry, blood pressure, NW7
Volume 4 Issue 3 - 2017

\author{
Akimichi Ohsaka \\ Department of Transfusion Medicine and Stem Cell Regulation, \\ Juntendo University School of Medicine, Japan
}

\begin{abstract}
Correspondence: Akimichi Ohsaka, MD, PhD, Department of Transfusion Medicine and Stem Cell Regulation, Juntendo University School of Medicine, 2-I - I Hongo, Bunkyo-ku, Tokyo I | 3-842 I, Japan, Fax +8 I-3-38 I I-2724, Tel +8 I-3-5802- I I09, Email ohsaka@juntendo.ac.jp
\end{abstract}

Received: March 26, 2017| Published: April 25, 2017
Abbreviations: SHOT, serious hazards of transfusion; IBCT, incorrect blood component transfused; RBC, red blood cell; PLT, platelet; FFP, fresh-frozen plasma; EIS, electronic identification system; PABD, preoperative autologous blood donation; JUH, juntendo university hospital; JSTMCT, Japan society of transfusion medicine and cell therapy; ATDUH, association of transfusion division of university hospitals; HPC, hematopoietic progenitor cell; UCB, umbilical cord blood; RFID, radiofrequency identification

\section{Introduction}

The risks of blood transfusion fall into several broad categories: immune and serologic transfusion reactions; blood-transmitted infections; lesser known complications, including transfusion-related acute lung injury, transfusion-associated circulatory overload and transfusion-associated graft-versus-host disease; and those associated with human error, especially mistransfusion. Traditionally, attention to increasing transfusion safety has focused mainly on the prevention of known viral transmission. The current risks of acquiring viral transmission through blood components and products are very small. ${ }^{1}$ Therefore, serious non-infectious hazards of transfusion have emerged as the most common complications. ${ }^{2}$ The risk of noninfectious complications, including risks related to hospital-based steps in transfusion care, is at least 100 times greater than the risk of acquiring human immunodeficiency virus or hepatitis $\mathrm{C}$ virus infection through blood components. ${ }^{3}$ One of the most frequent causes of transfusion-associated morbidity or mortality is mistransfusion. Mistransfusion, in which the wrong blood is transfused to the wrong patient, remains the most common type of error in transfusion practice. In particular, ABO-incompatible transfusions attributable to the incorrect identification of the patient or the blood unit are among the most serious of transfusion hazards. ${ }^{3-5}$ Mistransfusion is the final outcome of one or more procedural errors or technical failures in the transfusion process, starting with the decision to transfuse a patient and ending with the actual administration of blood components. ${ }^{3}$ The Japan Society of Transfusion Medicine and Cell Therapy (JSTMCT) conducted nationwide surveys in Japan regarding ABOincompatible blood transfusions (January 1995 to December 1999 in their first survey and January 2000 to December 2004 in their second survey) and found that the main cause of $\mathrm{ABO}$-incompatible transfusion was identification error between the patient and blood unit. ${ }^{6}$ In these surveys, the number of fatalities reported was nine and eight, respectively, being considered preventable. The prevention of identification check errors, regarding either the patient or blood unit, is of major importance in current transfusion practices and reducing the rate of human errors increases transfusion safety.

Sazama $^{4}$ described the issue of transfusion-associated deaths reported to the Food and Drug Administration from 1976 through 1985; in which $51 \%$ of 256 reported deaths resulted from acute hemolysis following the transfusion of ABO-incompatible blood products. Linden et al. ${ }^{5}$ calculated the risk of $\mathrm{ABO}$-incompatible transfusion to be 1 in 38,000 units and erroneous administration was observed for 1 of 19,000 red blood cell (RBC) units administered in New York State. The Serious Hazards of Transfusion (SHOT) scheme in England showed that, of 2,630 reported adverse events between 1996 and 2004, 1,832(70\%) were incorrect blood component transfused (IBCT) and the frequency of IBCT events was calculated as 7 per 100,000 components. ${ }^{7}$ Similar findings have been reported from the hemovigilance program in Quebec, in which ABO-incompatible transfusion was the most common major adverse event, occurring at a rate of 1 in 13,000 transfusions. ${ }^{8}$ However, the true incidence of 
mistransfusion seems to be even higher due to a failure to recognize many errors and because of a lack of complete denominator data on transfusion episodes. In this review, I present our own experiences regarding the role of electronic pre-transfusion check at the bedside in various settings.

\section{Discussion}

\section{Pre-transfusion check at the bedside in the hospital}

Background: The SHOT scheme showed that approximately $70 \%$ of IBCT event errors take place in clinical areas, with the most frequent error being failure of the final patient identification check at the bedside. ${ }^{7}$ Mistransfusion typically results from an error made during the pre-transfusion checking procedure just prior to blood administration, through which the blood unit is confirmed to be correct for the intended recipient. Pre-transfusion check at the bedside is the most critical step for the prevention of mistransfusion and represents the final opportunity to interrupt a misguided blood component. However, a large observational audit revealed a failure to perform the final bedside check, ${ }^{9}$ in which the practice compliance of healthcare workers for identification and vital sign monitoring of patients receiving blood transfusions were substandard in many hospitals. A bar code-based EIS is ideally suited to pre-transfusion checking requirements and has recently been reported to significantly improve transfusion practice. ${ }^{10-18}$ The SHOT scheme also reported that approximately $30 \%$ of errors pertaining to IBCT events occur in the hospital transfusion laboratory. ${ }^{7}$ These may involve the selection of the wrong sample for testing, transposition of labels, technical or transcription errors in manual pre-transfusion testing, or knowledgebased errors such as the selection of components not of an appropriate specification. This may also lead to a need to use an EIS in the hospital transfusion laboratory.

Transfusion practices in japan: Transfusion practices should be performed in the hospital in accordance with the transfusion policy, being approved by the hospital transfusion committee and including, in Japan, the Guidelines and Information for Using Blood Products and Blood Transfusion Therapy (Japan Guidelines), issued by the Ministry of Health, Labour and Welfare in Japan (September 2005, partially updated in March 2012). Japan Guidelines have recommended the issues regarding the pre-transfusion check procedures at the bedside that

i. Blood components should be preserved under their appropriate conditions

ii. The transfusionist (doctor or nurse) should check the appearance of blood components before initiating blood administration

iii. An arrangement and initiation of blood administration should be performed for only one patient

iv. For the prevention of mistransfusion attributable to clerical errors, the transfusionist should check the information regarding both the patient and blood unit, i.e., patient's name, blood group, product lot number, date of collection, results of compatibility testing and gamma irradiated or not for blood components

v. A standard two-person visual and verbal double-check should be performed regarding the above issues before initiating blood administration at the bedside and

vi. The transfusionist should check patient's vital signs, including body temperature, blood pressure, pulse and if possible $\mathrm{SpO}_{2}$ (oxygen saturation by pulse oximetry), before initiating blood administration. Finally, Japan Guidelines recommended the use of an EIS to ensure the pre-transfusion check at the bedside.

\section{The juntendo experience}

Pre-transfusion check procedures using an EIS: A bar code-based EIS (Nursing Pass, Bio-Rad Laboratories, Tokyo, Japan, previously Olympus Systems), linking the transfusion management system and hospital information system via a network, was implemented in JUH in July 2002. ${ }^{15}$ In Japan, the bar code-based EIS is based on the employment of the linear bar code (NW7), printed on labels attached to all allogeneic blood components supplied from branches of the Japanese Red Cross Blood Centre. The bar code on allogeneic blood components identifies the blood group, product type, unit of blood, product number depending on the donor and date of collection. The system is composed of:

i. A hand-held device incorporating a laser bar code scanner

ii. The patient's wristband with a bar code and eye-readable identification

iii. A wristband printer (Petit Lapin, Sato, Tokyo, Japan)

iv. An identification badge for staff with individual bar codes

v. A compatibility label imprinted with bar codes and

vi. A compatibility report form imprinted with bar codes.

All patients admitted to the hospital were given wristbands with a bar code and eye-readable identification that included the surname, first name, sex, date of birth, patient identification number and blood group $\mathrm{ABO} / \mathrm{RhD}$. The hand-held device is capable of reading bar codes during the verification procedures, receiving transfusion data via a network and sending data regarding bedside verification to the host computer in the transfusion service. In a previous publication, ${ }^{15}$ two different methods, i.e., wired and wireless, coexisted in the system, resulting in a difference of the bedside verification procedure between inpatient wards and operating rooms. Since February 2009, the EIS has been connected by wireless technology alone.

The transfusion policy at the bedside in JUH includes a standard two-person (mainly a doctor/nurse pair, occasionally two nurses) visual and verbal double-check first, followed by an electronic pretransfusion check using a hand-held device 'just' prior to the initiation of blood administration. Two verification procedures, i.e., pre-issuing and bedside, are separately carried out at the transfusion service and at the bedside, respectively. All blood components are delivered from the transfusion service after the completion of pre-issuing verification. Although pre-issuing verification does not play an essential role in the pre-transfusion checking process, it may contribute to enhancing the overall safety of transfusion. At the bedside, the transfusionist sequentially scans bar codes of his/her own identification badge, the patient's wristband and blood unit using a hand-held device (Figure 1A). In operating rooms, where a hand-held device is used for one patient until his/her operation has finished, electronic pre-transfusion check procedures are composed of two parts, i.e., entrance and bedside. In the entrance check procedure, a nurse scans the bar code of the patient's wristband using a hand-held device when the patient enters the operating room. In the bedside (operating table) check procedure, after completing two-person visual and verbal doublecheck, the transfusionist scans only the bar code of the blood with one scan for each unit just prior to initiating blood administration. If the bar codes of the wristband and blood are identical, the screen of the hand- 
held device displays ' $O K$ '. Non-matching data result in a warning of 'NG' with an alarm sound (Figure 1B). The match happens at the level of software installed in the hand-held device. After completing the electronic pre-transfusion check, the transfusionist 'immediately' initiates blood administration.
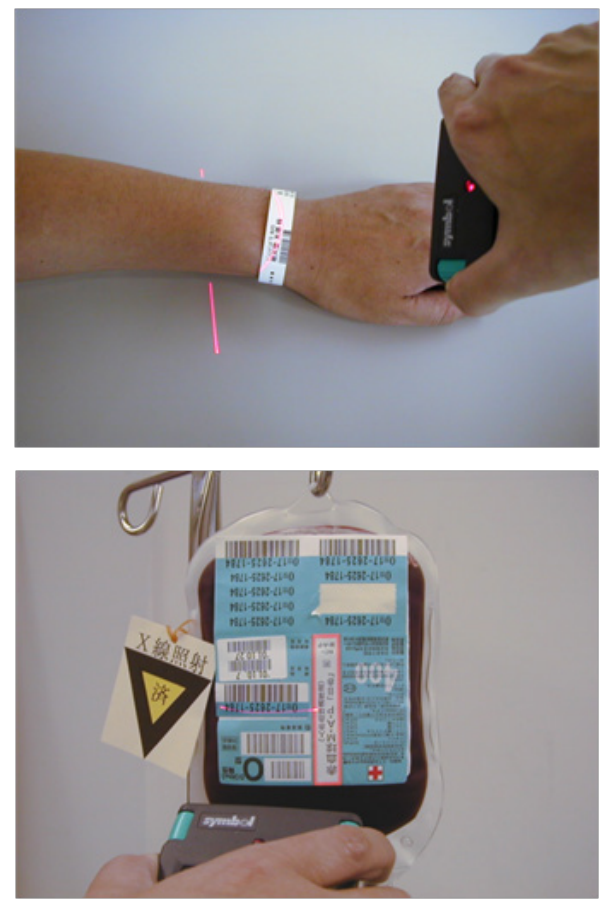

Figure IA At the bedside, after completing two-person visual and verbal double-check, the transfusionist sequentially scans bar codes of his/her own ID badge, the patient's wristband (upper panel) and the blood unit (lower panel) using a hand-held device.
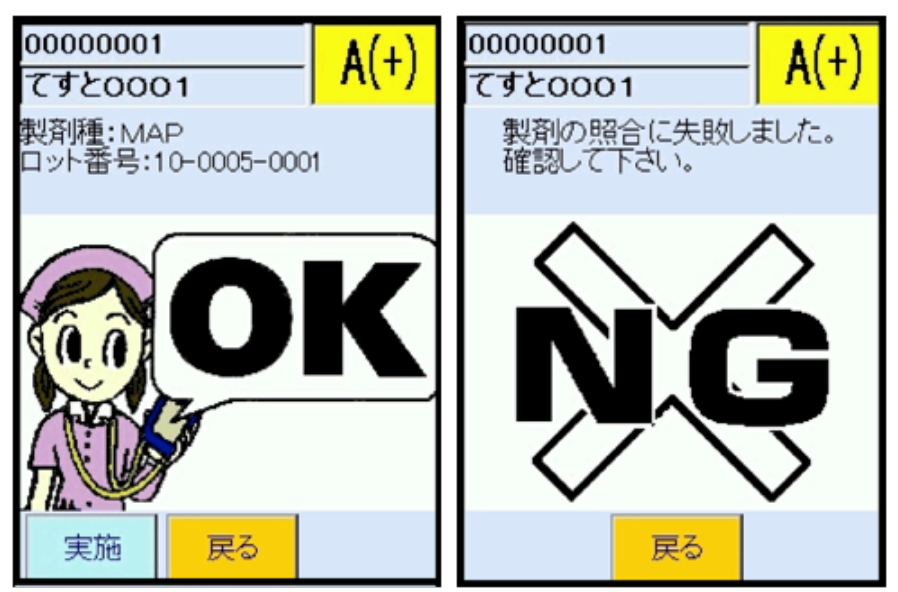

Figure IB Screen display from a hand-held device of the EIS showing the bedside verification procedure for confirmation that the blood unit is intended for the patient. Non matching data results in a warning "NG" with an alert sound.

Allogeneic blood transfusion: Between July 2002 and December 2012, a total of 114,855 blood components, including 63,255 RBCs, 21,586 platelets (PLTs) and 30,014 Fresh Frozen Plasma (FFP), were transfused without a single mistransfusion in JUH, of which 85,191 were administered on inpatient wards, 13,391 in outpatient units and 16,273 in operating rooms. The number of blood components administered with a standard two-person visual and verbal double check but without using the EIS was 2,122 overall, 1,490 on inpatient wards, 169 in outpatient units and 463 in operating rooms. Thus, the compliance rate with the electronic pre-transfusion check at the bedside was $98.2 \%$ overall, $98.3 \%$ on inpatient wards, $98.7 \%$ in outpatient units and $97.2 \%$ in operating rooms. ${ }^{19}$

Causes of failure of electronic pre-transfusion check: We previously reported the causes of failure of the 'second' electronic pre-transfusion check at the bedside. ${ }^{20}$ All errors leading to the failure of electronic pre-transfusion check were categorized into four broad groups: human, hand-held device, system and wristband errors. Human errors were further divided into manipulation and neglectbased errors. The categorization and definition of errors leading to the failure of electronic pre-transfusion check are described in Table 1. The compliance rate with electronic pre-transfusion check at the time of the study was $97.8 \% .{ }^{20}$ The cause of failure of electronic pre-transfusion check was human error in 811 cases $(84.7 \%)$, hand-held device error in 74(7.7\%), system error in 50(5.2\%) and wristband error in $23(2.4 \%)$. Of the human errors, $698(86.1 \%)$ were manipulation errors and the remaining $113(13.9 \%)$ were neglectbased errors. Manipulation errors mainly decreased after the initiation of notification, where the transfusion service notified at one hour after the issuing of blood components to clarify the cause of failure of an electronic pre-transfusion check and neglect-based errors were still reported. The mean number of human, manipulation and neglectbased errors significantly decreased after the initiation of notification. These errors may be decreased by the further education and training of medical staff and continued support from the transfusion service.

Table I Categorization and definition of errors leading to failure of the 'second' electronic pre-transfusion check at the bedside

\begin{tabular}{ll}
\hline Category & Definition \\
\hline Human errors & \\
$\begin{array}{ll}\text { Manipulation } & \text { The transfusionist did not manipulate the hand-held } \\
\text { errors } & \text { device correctly }\end{array}$
\end{tabular}

Neglect-based The transfusionist did not act in accordance with the errors transfusion policy*

Hand-held Failures of the device itself, including battery failure device errors or it being dropped

System errors communication, including down- or up-loading errors using hand-held devices

$\begin{array}{ll}\text { Wristband } & \text { Failures of the patient's wristband, where patients } \\ \text { errors } & \text { were not wearing them at the time of transfusion } \\ \text { because they were intentionally removed }\end{array}$

*The transfusion policy in JUH includes a standard two-person visual and verbal double-check that should be performed first, followed by electronic pretransfusion check using a hand-held device just prior to blood administration. In this case, the 'second' electronic pre-transfusion check at the bedside might be omitted due to urgency or time constraints. EIS, electronic identification system.

Preoperative autologous blood donation and transfusion: $\mathrm{PABD}$ involves collecting and storing the patient's own blood prior to surgery in order to administer it, if necessary, post-operatively. It has been advocated to reduce the risk related to allogeneic blood transfusion. However, PABD transfusion is associated with risks. One $(0.006 \%)$ in 16,783 autologous donations has been reported to be associated with an adverse reaction severe enough to require hospitalization, ${ }^{21}$ which is nearly 12 times the risk associated with allogeneic donation. 
This difference is probably attributable to the fact that autologous blood donors have underlying pathologic conditions such as cardiac, vascular and respiratory disease. The transfusion of autologous blood involves many of the same complications as that of allogeneic units, such as the risk of administrative errors. Patients may receive another patient's autologous blood as a result of clerical errors or may receive allogeneic blood when autologous blood is available. There have been few studies examining the frequency of errors in PABD transfusion. Ohto et al. $^{22}$ reported a nationwide survey in Japan regarding autologous blood donation and transfusion and identified 117 errors or problems $(0.47 \%)$ in 24,929 PABD transfusions, of which one unit $(0.004 \%)$ was administered to the wrong patient. Domen ${ }^{23}$ reported a total of $20(0.072 \%)$ adverse reactions in 27,859 autologous blood transfusions at the Cleveland Clinic, of which one $(0.0054 \%)$ in 18,506 intra-operatively salvaged blood products, were transfused to the wrong patient. Because the error rate of autologous and allogeneic blood transfusions appears to be similar, autologous transfusion should be approached with the same level of care and consideration as allogeneic transfusion.

A bar code-based EIS was applied to the pre-transfusion check procedure in the setting of PABD transfusion in JUH. ${ }^{24}$ Compatibility labels were affixed to PABD units, on which an in-house bar code identifying the product type and number was printed, making it possible to perform electronic pre-transfusion check at the bedside using hand-held devices. In this study, autologous blood transfusion did not include acute normovolemic hemodilution or intra-operative cell salvage. Of 7,419 PABD units collected, a total of 5,627(76\%) units, including 3,243 RBC, 1,847 FFP and 537 cryoprecipitate units, were administered without a single mistransfusion. The compliance rate with the electronic pre-transfusion check at the bedside for PABD transfusion was $99 \%$. Human error was the most frequent cause of errors leading to the failure of 'second' electronic pre-transfusion check. Comparing to allogeneic blood transfusion, the relative rate of neglect-based errors in PABD units $(43 \%)$ was considerably higher than that in allogeneic blood units (14\%). The reason for the higher rate of neglect-based errors in PABD transfusion remains unknown. Because $75 \%$ of PABD units were administered in operating rooms, emergent or time-constrained situations would seem to cause neglect of the transfusion policy.

Pediatric transfusion: Because of differences in the age, size and maturity of physiologic systems, children undergoing transfusion may incur different risks compared to adults. A multi-institutional analysis of blood transfusion has been reported in a large cohort of pediatric patients not more than 18 years of age, in which the overall complication rate in the study was 10.7 per 1,000 transfusions. ${ }^{25}$ The complication rate in adults has been reported to be 2.5 per 1,000 transfusions in a national incident reporting system of France. ${ }^{26}$ The SHOT scheme ${ }^{27}$ showed the estimation of the incidence of an adverse outcome to be 18:100,000 transfusions for children less than 18 years and 37:100,000 for infants less than 12months, compared to $13: 100,000$ for adults. The SHOT classifies incidents reported into 12 different categories; ${ }^{28}$ of these, IBCT event errors were the most common category in both adults and children, but they were particularly common in children, making up more than $80 \%$ of reports. ${ }^{26}$ These findings suggest that pediatric patients are more vulnerable to transfusion-associated complications than adults. Pediatric transfusion has specific requirements and the SHOT subcategory of IBCT event errors including special requirements not met, i.e., CMV-negative blood, irradiated blood. There are further requirements regarding the transfusion of blood components, including small-volume transfusions, dispensing blood in plastic syringes and infusion through small-gauge needles with or without a mechanical infusion pump. Many transfusion services use only RBC components for infants less than 4 months, because they are unlikely to develop acute hemolytic transfusion reactions. However, a safety strategy for preventing mistransfusion is needed in pediatric patients being transfused blood components of PLTs or FFP. Administrative error is also a SHOT subcategory of the IBCT event errors and there are a number of unique problems seen in children with regard to identification. This may also lead to a need for the use of an EIS in pediatric transfusion.

A bar code-based EIS was applied to the pre-transfusion check procedure in the setting of pediatric transfusion in $\mathrm{JUH}^{29}$ Blood dispensed in syringes was attached to compatibility labels, on which the bar code of the primary bag of blood was printed, making it possible to perform electronic pre-transfusion check using handheld devices. Wristbands were occasionally attached to the patient's ankle or nameplate at the bedside in cases of neonates and preterm infants. In this study, a total of $3,957(10 \%$ of all transfusions) blood components were administered to pediatric patients without a single mistransfusion, of which $871(22 \%$ of pediatric transfusions $)$ were issued by dispensing in syringes and the remaining 3,086(78\%) were issued in bags. The compliance rate with the electronic pre-transfusion check at the bedside was $99 \%$ in blood dispensed in syringes. The cause of failure of electronic pre-transfusion check was human error in all cases.

Application of EIS for blood transfusion into the setting of hematopoietic progenitor cell infusion at the bedside: Intensive chemotherapy followed by HPC transplantation has widely been used in the treatment of certain hematological diseases and solid tumors. Among the transplant procedures, the infusion of HPC components at the bedside is a critical step for the success in HPC transplantation and the failing to receive appropriate HPCs for the recipient after intensive chemotherapy can be fatal. ${ }^{30}$ However, mislabeled units of umbilical cord blood (UCB) from highly reputable UCB banks have been reported. ${ }^{31}$ In addition, errors in mislabeling of deceased donor kidneys have been reported. ${ }^{32}$ Mislabeling of grafts may lead to a fatal outcome in any transplantation. Although we had no experience of an incorrect infusion of the HPC component to the recipient in JUH, we implemented a bar code-based EIS for blood transfusion into the setting of HPC transplantation.

A bar code-based EIS for blood transfusion was applied into the setting of HPC transplantation in July 2003. In the case of HPC components, in-house bar codes identifying both the patient's and component's details are attached. This step is critical for the management of HPC components in the transfusion service. Peripheral blood HPC components collected in the hospital are linked to the patient's information when the components are prepared and managed in the transfusion service. Whereas HPC components from banks are not specified to the patient in the hospital, they have to be correctly managed using, at least, a bar code-based technology in the transfusion service, as blood units. The data include the patient's identification number, surname, first name, sex, date of birth and blood group; and the component's type and lot number. All HPC components are delivered from the transfusion service after completing the electronic pre-issuing check. At the bedside, after completing two-person visual and verbal double-check, the infusionist sequentially scans bar codes of his/her own identification badge, the patient's wristband and the HPC bag using a hand-held device. After completing the electronic 
pre-infusion check, the infusionist immediately initiates HPC infusion at the bedside. In this study, a total of 518 HPC components were infused to 190 patients without a single misinfusion in the hospital. An overall compliance rate with the electronic pre-infusion check at the bedside was $100 \%$. Among specialities, hematology (77\%) was most frequently required HPC infusion, followed by pediatrics $(21 \%)$ and urology (2\%). The purpose of the use of an EIS is to avoid human errors and to ensure the infusion of the right HPC bag to the right patient. Pre-infusion check at the bedside is the most critical step for the prevention of misinfusion of HPC components to the patient.

\section{Recommendations for the electronic pre-transfusion} check at the bedside

\section{Abstract of the Guidelines}

Background: JSTMCT Working Party on hemovigilance and safety management of blood transfusion issued the first version of guidelines for the electronic pre-transfusion check for blood administration at the bedside. ${ }^{33}$ The current guidelines cover issues concerning:

i. Pre-transfusion check procedures at the bedside

ii. Requirements for the electronic pre-transfusion check at the bedside

iii. Pre-transfusion check procedure at the bedside for a conscious patient

iv. Pre-transfusion check procedure at the bedside for an unconscious patient or child

v. Pre-issuing check procedure at the transfusion service and

vi. Monitoring of the bedside use of the issued blood components.

When an EIS is implemented in a hospital, the pre-transfusion check procedures at the bedside may involve one or two healthcare professionals. When one individual carries out the pre-transfusion check procedure using an EIS, it seems to be plausible. However, if electronic pre-transfusion check at the bedside fails due to human error, ${ }^{20}$ one-person bedside checking without new technology may present a higher risk of mistransfusion than a standard two-person double-check, although the number of people required checking the identity of the patient and blood unit at the bedside is controversial. ${ }^{34}$ Japan Guidelines recommended a standard two-person visual and verbal double-check for pre-transfusion check procedure at the bedside. In addition, the recent BCSH Guidelines stated that "the use of a bedside blood tracking system does not replace the role of the well trained and competency assessed clinician who administers blood components. ${ }^{35}$ " The current guidelines do not positively recommend a one-person pre-transfusion check at the bedside even if using an EIS. Thus, the current guidelines recommend that the electronic pre-transfusion check procedures at the bedside should be basically carried out by 'two-person', of whom one should be the transfusionist and the other should be the second checker. The second checker may not be limited to healthcare professionals, such as the patient. When the patient is conscious the transfusionist (nurse or doctor) together with the patient, conducts the pre-transfusion check procedures at the bedside using an EIS. When the patient is unconscious or child, two nurses (or a doctor/nurse pair) conduct the pre-transfusion check procedures as well. In this case, another healthcare professional, such as the staff member of the transfusion service who delivered the issued blood component into the clinical area, instead of the patient, may be available for the second checker.
Pre-transfusion check procedure at the bedside for a conscious patient: This case may be a common situation in most hospitals. The entire process of the electronic pre-transfusion check procedures for a conscious patient should be conducted basically by one nurse (or doctor), together with the patient and carried out at the bedside using an EIS. The role of the patient is expected to act as the second checker. Thus, another healthcare professional may also be available. The transfusionist:

i. Asks to the patient to state his/her full name and date of birth.

ii. Checks the clerical information, together with the patient, under reviewing both the blood unit and the compatibility report form.

iii. Scans sequentially the bar codes of his/her own identification badge, the patient's wristband and the blood unit using the hand-held device.

iv. Verifies the displaying data on the hand-held device, together with the patient, where the bar codes match or not between the wristband and the blood unit.

v. Initiates 'immediately' blood administration if the hand-held device displays ' $O K$ '.

Pre-transfusion check procedure at the bedside for an unconscious patient or child: This case may be a common situation in the intensive care unit, emergency rooms and on inpatient wards for children. The entire process of the electronic pre-transfusion check procedures for an unconscious patient or child should be conducted basically by two nurses (or a doctor/nurse pair) and carried out at the bedside using an EIS. If two nurses conduct the electronic pre-transfusion check procedures at the bedside, one should act as the transfusionist and the other as the second checker. In the case of a doctor/nurse pair, a nurse may act as the transfusionist and a doctor as the second checker. The second checker may also be available for another healthcare professional. The transfusionist:

i. Checks the patient's full name and date of birth, together with the second checker, under reviewing the patient's wristband.

ii. Checks the clerical information, together with the second checker, under reviewing both the blood unit and the compatibility report form.

iii. Scans sequentially the bar codes of his/her own identification badge, the patient's wristband and the blood unit using the hand-held device.

iv. Verifies the displaying data on the hand-held device, together with the second checker, where the bar codes match or not between the wristband and the blood unit.

v. Initiates 'immediately' blood administration if the hand-held device displays ' $\mathrm{OK}$ '.

In regards to the detailed descriptions, please read the original manuscript. ${ }^{33}$ The first version of guidelines for the electronic pretransfusion check for blood administration at the bedside has been established, being appropriate for the clinical situation in Japan, because approximately $90 \%$ of Japanese teaching hospitals have implemented an EIS for pre-transfusion check procedures.

\section{Prevalence of an EIS in japan}

The Association of Transfusion Division of University Hospitals 
(ATDUH, 2014, chairman Teruhisa Fujii, Hiroshima University Hospital, Hiroshima, Japan) made inquiries using questionnaires involving a small cohort of the transfusion service of university hospitals in Japan $(n=91)$. The members of ATDUH are teaching hospitals and representatives of regions distributed widely in Japan and also those of JSTMCT. All the transfusion service in university hospitals was registered. The questions referred to the transfusion practices and use of IT systems, including a transfusion management system and a bar code-based EIS: vendor of the system, name of application for the system, date of initiation of the system and compliance of the electronic pre-transfusion check at the bedside. Of the questionnaires sent to 91 transfusion services, 90(99\%) were returned fully completed. Finally, 81(90\%) transfusion services answered that the pre-transfusion check procedures have been carried out using a bar code-based EIS. At present, the overall prevalence rate of an EIS for pre-transfusion check procedures at the bedside in Japanese university hospitals is $90 \%$. Further studies including a nationwide survey are needed to explore the prevalence of an EIS in Japan.

\section{Conclusion}

Bar code technology is a widely used, reliable and inexpensive machine-readable identification system. Because the Japanese Red Cross Blood Centre supplies all allogeneic blood components with attached labels containing linear bar codes, the EIS presently employs linear bar codes in Japan. It has been reported that bar code-related patient misidentifications occur when a linear bar code is used. ${ }^{36}$ More advanced systems such as radiofrequency identification (RFID) will be introduced in the near future. RFID may have advantages over bar code-based technology, i.e., more user-friendly, facilitating the recording of more information. Technology-based solutions designed to prevent mistransfusion have recently been developed and the effectiveness of the different systems in detecting errors has been reported. ${ }^{37}$ The economic aspects and availability of products remain issues regarding implementing these technologies. To reduce human error and the risk of mistransfusion, we have to address the issue at the hospital level, employing a system-based approach.

\section{Acknowledgements}

None.

\section{Conflict of interest}

The author declares no conflict of interest.

\section{References}

1. Gonzalez M, Règine V, Piccinini V, et al. Residual risk of transfusiontransmitted human immunodeficiency virus, hepatitis $\mathrm{C}$ virus, and hepatitis B virus infections in Italy. Transfusion. 2005;45(10):1670 1675 .

2. Hendrickson JE, Hillyer CD. Noninfectious serious hazards of transfusion. Anesth Analg. 2009;108(3):759-769.

3. Dzik WH. Emily Cooley lecture 2002: transfusion safety in the hospital. Transfusion. 2003;43(9):1190-1199.

4. Sazama K. Reports of 355 transfusion-associated deaths: 1976 through 1985. Transfusion. 1990;30(7):583-590.

5. Linden JV, Wagner K, Voytovich AE, et al. Transfusion errors in New York State: an analysis of 10years' experience. Transfusion. 2000;40(10):1207-1213.
6. Fujii Y, Shibata Y, Miyata S, et al. Consecutive national surveys of ABOincompatible blood transfusion in Japan. Vox Sang. 2009;97(3):240-246.

7. Stainsby D, Jones H, Asher D, et al. Serious hazards of transfusion: a decade of hemovigilance in the UK. Transfus Med Rev. 2006;20(4):273282.

8. Robillard P, Itaj NK, Corriveau P. ABO incompatible transfusions, acute and delayed hemolytic transfusion reactions in Quebec hemovigilance system. Transfusion. 2002;42:25S.

9. Novis DA, Miller KA, Howanitz PJ, et al. Audit of transfusion procedures in 660 hospitals. A College of American Pathologists Q-Probes study of patient identification and vital sign monitoring frequencies in 16494 transfusions. Arch Pathol Lab Med. 2003;127(5):541-548.

10. Jensen NJ, Crosson JT. An automated system for bedside verification of the match between patient identification and blood unit identification. Transfusion. 1996;36(3):216-221.

11. Turner CL, Casbard AC, Murphy MF. Barcode technology: its role in increasing the safety of blood transfusion. Transfusion. 2003;43(9):1200 1209.

12. Miyata S, Kawai T, Yamamoto S, et al. Network computer-assisted transfusion-management system for accurate blood component-recipient identification at the bedside. Transfusion. 2004;44(3):364-372.

13. Chan JC, Chu RW, Young BW, et al. Use of an electronic barcode system for patient identification during blood transfusion: 3 -year experience in a regional hospital. Hong Kong Med J. 2004;10(3):166-171.

14. Askeland RW, McGrane S, Levitt JS, et al. Improving transfusion safety: implementation of a comprehensive computerized bar codebased tracking system for detecting and preventing errors. Transfusion. 2008;48(7):1308-1317.

15. Ohsaka A, Abe K, Ohsawa T, et al. A computer-assisted transfusion management system and changed transfusion practices contribute to appropriate management of blood components. Transfusion. 2008;48(8):1730-1738.

16. Bennardello F, Fidone C, Cabibbo S, et al. Use of an identification system based on biometric data for patients requiring transfusions guarantees transfusion safety and traceability. Blood Transfus. 2009;7(3):193-203.

17. Pagliaro P, Turdo R, Capuzzo E. Patients' positive identification systems. Blood Transfus. 2009;7(4):313-318.

18. Uríz MJ, Antelo ML, Zalba S, et al. Improved traceability and transfusion safety with a new portable computerised system in a hospital with intermediate transfusion activity. Blood Transfus. 2011;9(2):172-181.

19. Ohsaka A, Ohsawa T, Nakamura Y, et al. Evaluation of compliance with electronic pretransfusion check for blood administration at the bedside: a 10·5-year experience at a university hospital. VOXS. 2015;10(2):65-72.

20. Ohsaka A, Kobayashi M, Abe K. Causes of failure of a barcode-based pre-transfusion check at the bedside: experience in a university hospital. Transfus Med. 2008;18(4):216-222.

21. Popovsky MA, Whitaker B, Arnold NL. Severe outcomes of allogeneic and autologous blood donation: frequency and characterization. Transfusion. 1995;35(9):734-737.

22. Ohto H, Fuji T, Wakimoto N, et al. A survey of autologous blood collection and transfusion in Japan in 1997. Transfus Sci. 2000;22(12):13-18.

23. Domen RE. Adverse reactions associated with autologous blood transfusion: evaluation and incidence at a large academic hospital. Transfusion. 1998;38(3):296-300.

24. Ohsaka A, Furuta Y, Ohsawa T, et al. Bar code-based pre-transfusion check in pre-operative autologous blood donation. Transfus Apher Sci. 2010;43(2):183-188. 
25. Slonim AD, Joseph JG, Turenne WM, et al. Blood transfusions in children: a multi-institutional analysis of practices and complications. Transfusion. 2008;48(1):73-80.

26. Andreu G, Morel P, Forestier F, et al. Hemovigilance network in France: organization and analysis of immediate transfusion incident reports from 1994 to 1998. Transfusion. 2002;42(10):1356-1364.

27. Stainsby D, Jones H, Wells AW, et al. Adverse outcomes of blood transfusion in children: analysis of UK reports to the serious hazards of transfusion scheme 1996-2005. Br J Haematol. 2008;141(1):73-79.

28. Harrison E, Bolton P. Serious hazards of transfusion in children (SHOT) Paediatr Anaesth. 2011;21(1):10-13.

29. Ohsaka A, Abe K, Nakamura Y, et al. Issuing of blood components dispensed in syringes and bar code-based pretransfusion check at the bedside for pediatric patients. Transfusion. 2009;49(7):1423-1430.

30. Heilborn AS, Kadidlo D, McCullough J. Patient care during infusion of hematopoietic progenitor cells. Transfusion. 2004;44(6):907-916.

31. McCullough J, McKenna D, Kadidlo D, et al. Mislabeled units of umbilical cord blood detected by a quality assurance program at the transplantation center. Blood. 2009;114(8):1684-1688.
32. Friedman AL, Lee KC, Lee GD. Errors in ABO labeling of deceased donor kidneys: case reports and approach to ensuring patient safety. $\mathrm{Am}$ J Transplant. 2007;7(2):480-483.

33. Ohsaka A, Kato H, Kino S, et al. Recommendations for the electronic pre-transfusion check at the bedside. Blood Transfus. 2016;14(5):419424.

34. Watson D, Murdock J, Doree C, et al. Blood transfusion administrationone- or two-person checks: which is the safest method? Transfusion. 2008;48(4):783-789.

35. Jones J, Ashford P, Asher D, et al. Guidelines for the specification, implementation and management of information technology systems in hospital transfusion laboratories. Transfus Med. 2014;24(6):341-371.

36. Snyder ML, Carter A, Jenkins K, et al. Patient misidentifications caused by errors in standard bar code technology. Clin Chem. 2010;56(10):15541560 .

37. Pagliaro P, Rebulla P. Transfusion recipient identification. Vox Sang. 2006;91(2):97-101. 